\title{
Structural Basis of p97 Inhibition by the Site-Selective Anticancer Compound CB-5083
}

\author{
Wai Kwan Tang, Taivan Odzorig, Whitney Jin, and Di Xia \\ Laboratory of Cell Biology, Center for Cancer Research, National Cancer Institute, National Institutes of Health, \\ Bethesda, Maryland
}

Received September 10, 2018; accepted December 20, 2018

\section{ABSTRACT}

Inhibition of p97, a key player in the ubiquitin-proteasome degradation pathway, has been proposed as a treatment of cancer. This concept was nearly realized recently when a potent p97 inhibitor, 1-[4-(benzylamino)-5H,7H,8H-pyrano[4,3d]pyrimidin-2-yl]-2-methyl-1H-indole-4-carboxamide (CB5083), was developed and demonstrated broad antitumor activity in various tumor models. CB-5083 functions as a competitive inhibitor that binds selectively to the ATP-binding site of the D2 domain, although both the D1 and D2 ATPase sites of p97 are highly similar. Despite its promising anticancer activity, CB-5083 failed its phase I clinical trials due to an unexpected off-target effect, which necessitates further improvement of the inhibitor. In this study, we determined the crystal structure of $\mathrm{N}$-terminal domain-truncated p97 in complex with CB-5083. It provides a structural basis for the specificity of CB-5083 toward the D2 domain, offers an explanation in atomic detail for the mutations that confer resistance to CB-5083, and establishes a foundation for future structure-guided efforts to develop the next generation of p97 inhibitors.

\section{Introduction}

Targeting nononcogene pathways for cancer treatment has recently gained much attention because of the rise of resistance as a result of oncogene-targeted therapies (Kenny et al., 2017). One of the nononcogene pathways being targeted is the ubiquitin-proteasome system (UPS); the relevance of interfering with the UPS has been proven in clinical settings by the success of proteasome inhibitors for the treatment of multiple myeloma (Scalzulli et al., 2018). However, the development of resistance against (Kale and Moore, 2012) and the lack of activity in solid tumor settings (Milano et al., 2009) of the proteasome inhibitors support the need to develop inhibitors of other regulators of cellular protein homeostasis.

Valosin-containing protein, also known as p97 in mammals and cdc48 in yeast, is a member of the broad AAA family of proteins: ATPases associated with various cellular activities. p97 serves many different cellular functions (Xia et al., 2016), one of which is critical regulation of protein homeostasis pathways such as the endoplasmic reticulum-associated degradation, which is the main quality control mechanism for soluble, membrane-associated, glycosylated as well as nonglycosylated proteins as they are processed through the endoplasmic reticulum (Ye et al., 2001). Mutations in the gene of $\mathrm{p} 97$ have been linked to degenerative disorders named

This research was supported by the Intramural Research Program of the National Institutes of Health, National Cancer Institute, and Center for Cancer Research.

https://doi.org/10.1124/mol.118.114256. multisystem proteinopathy 1 , previously known as inclusion body myopathy with Paget's disease of bone and frontotemporal dementia (IBMPFD) (Taylor, 2015; Tang and Xia, 2016).

Inhibition of $\mathrm{p} 97$ has been shown to lead to cell death mediated mainly by the unfolded protein response (Wang et al., 2008, 2009), a pathway that acts both to resolve unfolded protein stress and to trigger cell death when the buildup of such unfolded proteins becomes irresolvable (Ghosh et al., 2014). Demonstrated in vitro as a molecular unfoldase (Beskow et al., 2009; Blythe et al., 2017; Bodnar and Rapoport, 2017), p97 is thought to function in vivo as a powerful extractor of proteins from complex molecular machines and various organelles. Indeed, the molecular makeup of p97 supports this proposed function. p97 is a molecular engine powered by six ATP-hydrolyzing subunits and each subunit consists of an $\mathrm{N}$-terminal domain ( $\mathrm{N}$ domain) and two tandem ATPase domains (D1 and D2) (Fig. 1A), qualifying it as a type II AAA ATPase. Both the D1 and D2 domains are capable of hydrolyzing ATP, although the D2 domain contributes more to the overall ATPase activity of p97 (Ye et al., 2003; Tang and Xia, 2013).

The essential role of p97 in the UPS led to the hypothesis that by inhibiting the activity of p97, it may be possible to circumvent the resistance encountered subsequent to the use of proteasome inhibitors. One p97 inhibitor, 1-[4-(benzylamino)-5H,7H,8H-pyrano[4,3-d]pyrimidin-2-yl]-2-methyl-1Hindole-4-carboxamide (CB-5083) (Fig. 1B), was developed as a result of an extensive lead-optimization effort, tracing its origin to $\mathrm{DBeQ}$, a quinazolin-based p97 inhibitor (Chou and Deshaies, 2011; Chou et al., 2013). CB-5083 has been shown to be 
A

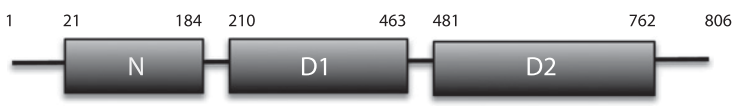

B

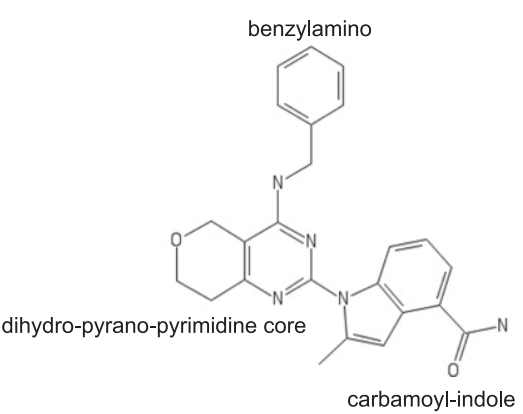

D

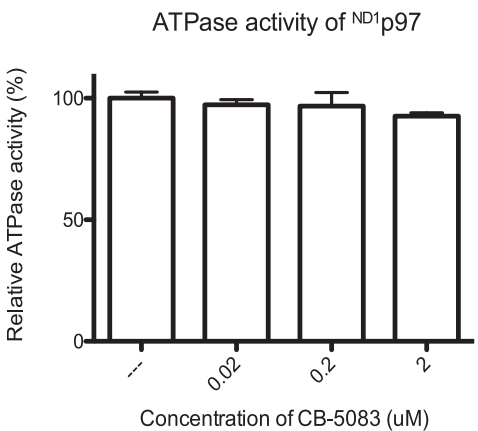

C

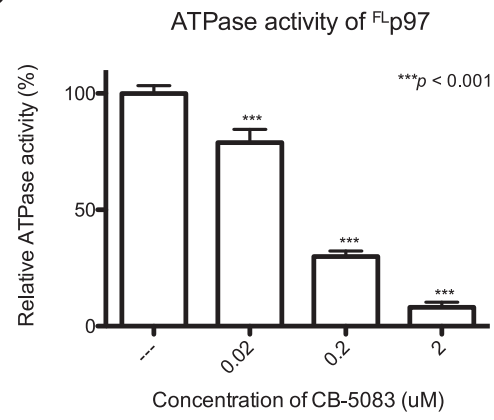

E

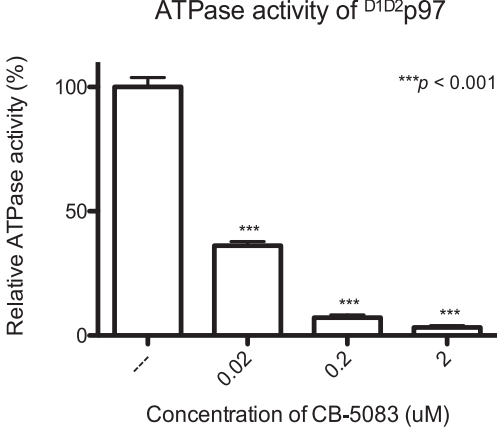

Fig. 1. Inhibition of p97 variants by CB-5083. (A) Domain organization of p97. (B) Chemical structure of CB-5083. Inhibition profiles of ${ }^{\mathrm{FL}} \mathrm{p} 97$ (C), ${ }^{\mathrm{ND} 1} \mathrm{p} 97$ (D), and ${ }^{\mathrm{D} 1 \mathrm{D} 2} \mathrm{p} 97$ (E) by CB-5083 at different concentrations. The ATPase activities are shown as mean \pm S.D. values from three technical replicates. Statistical significant differences ( $P$ value) between the presence and absence of inhibitor were analyzed by one-way analysis of variance. selective, specifically inhibiting the ATPase of the D2 domain of p97. Treatment of tumor cells with CB-5083 leads to accumulation of polyubiquitinated proteins and retention of endoplasmic reticulum-associated degradation substrates. This antitumor activity of CB-5083 is also exhibited in multiple myeloma, B acute lymphoblastic leukemia, and hematologic and solid tumor models by activating the apoptotic arm of the UPS (Anderson et al., 2015; Bastola et al., 2017; Gugliotta et al., 2017; Gareau et al., 2018). In preclinical studies, CB-5083 also showed auspicious results against proteasome inhibitorresistant multiple myeloma patient samples (Le Moigne et al., 2017).

Based on the promising results from preclinical studies, CB-5083 was advanced to phase I clinical trials to treat patients with lymphoid hematologic malignancies (CLC-102) and advanced solid tumors. However, the trials experienced setbacks and were terminated due to cytotoxicity arising from an unexpected offtarget effect (https://www.bizjournals.com/sanfrancisco/news/2018/ 05/31/can-viagra-help-revive-cleave-biosciences-biotech.html), suggesting that better, more selective p97 inhibitors are needed. As expected, the use of CB-5083 in laboratory settings quickly led to resistance, which has been shown to be largely a consequence of target site mutations (Anderson et al., 2015; Bastola et al., 2017). Disappointingly, not all resistance-conferring mutations confirm various models of CB-5083 binding to p97, which were created based on molecular docking experiments (Anderson et al., 2015; Bastola et al., 2017) and need to be replaced preferably by a more accurate experimental model. In this work, we report such a model, obtained by X-ray crystallography, for the interactions of CB-5083 with p97, which forms the basis for our interpretations of available mutational data. Additionally, this model offers a structural basis for the D2 domain selectivity, and more importantly provides guidance for future endeavors to develop more specific p97 inhibitors.

\section{Materials and Methods}

Protein Expression and Purification. Expression and purification of p97 were carried out as previously described (Tang et al., 2010). Variants of p97 containing different lengths $\left[{ }^{\mathrm{FL}} \mathrm{p} 97\right.$ (residues $1-806$ ), ${ }^{\mathrm{ND} 1} \mathrm{p} 97$ (residues 1-481), and ${ }^{\mathrm{D} 1 \mathrm{D} 2} \mathrm{p} 97$ (residues 210-806)] or mutations ( $^{\mathrm{FL}} \mathrm{p} 97^{\mathrm{V} 474 \mathrm{~S}},{ }_{\mathrm{FL}} \mathrm{p} 97^{\mathrm{I} 479 \mathrm{~S}},{ }_{\mathrm{FL}} \mathrm{p} 97^{\mathrm{L} 526 \mathrm{~S}}$, and $\left.{ }^{\mathrm{FL}} \mathrm{p} 97^{\mathrm{I656S}}\right)$ were generated using the Quikchange Site-Directed Mutagenesis Kit (Agilent Technologies, Santa Clara, CA).

Determination of ATPase Activity. The ATPase activity of p97 was determined by measuring the amount of inorganic phosphate released from ATP hydrolysis, which reacts with a complex of molybdate and malachite green (Hess and Derr, 1975; Lanzetta et al., 1979). The activity assay was performed in an assay buffer containing $50 \mathrm{mM}$ Tris-HCl, $\mathrm{pH} 8.0,20 \mathrm{mM} \mathrm{MgCl}_{2}$, and $1 \mathrm{mM}$ EDTA. A total of $50 \mu \mathrm{l}$ reaction mix containing $5 \mu \mathrm{g}$ of protein and $4 \mathrm{mM}$ ATP in the assay buffer was incubated at $37^{\circ} \mathrm{C}$ for $10-15$ minutes. The reaction was immediately stopped by the addition of $800 \mu$ l dye buffer (a fresh mixture of $0.045 \%$ malachite green and $1.4 \%$ ammonium molybdate tetrahydrate in $4 \mathrm{~N} \mathrm{HCl}$ in a 1:3 ratio) followed by the addition of $100 \mu \mathrm{l}$ of $34 \%$ sodium citrate solution after 1 minute. After 10-minute incubation at room temperature, $16 \mu \mathrm{l}$ of $10 \%$ Tween-20 
was added to dissolve any precipitate. Absorbance was then measured at $660 \mathrm{~nm}$. The amount of inorganic phosphate released was calculated based on a standard curve established by a known amount of $\mathrm{KH}_{2} \mathrm{PO}_{4}(50-300 \mu \mathrm{M})$ in assay buffer.

Crystallization and Structure Determination. Crystals of ${ }^{\text {D1D2 }}$ p97 in complex with CB-5083 were grown using the sitting-drop vapor diffusion method at $16^{\circ} \mathrm{C}$. A protein solution of $14 \mathrm{mg} / \mathrm{ml}$ was mixed with CB-5083 (ActiveBiochem, Kowloon, Hong Kong) (100 mM in dimethylsulfoxide) to reach a final concentration of $500 \mu \mathrm{M}$, and incubated overnight at $4^{\circ} \mathrm{C}$ with mixing. The admixture was then spun at $14,000 \mathrm{rpm}$ for 30 minutes at $4^{\circ} \mathrm{C}$. The supernatant was mixed with a well solution containing $0.1 \mathrm{M}$ sodium citrate, $\mathrm{pH}$ 4.5, $250 \mathrm{mM}$ Trisodium citrate, and $15 \%$ polyethylene glycol 3350 in a 1:1 ratio for crystallization. Crystals were cryo-protected first with the well solution followed by the same well solution supplemented with $19 \%$ polyethylene glycol 3350 and 10\% glycerol, and flash-cooled in liquid nitrogen.

X-ray diffraction experiments were carried out at the SER-CAT and GM/CA beam lines of the Advanced Photon Source at Argonne National Laboratory (Lemont, IL) at 100K. Diffraction images were processed and scaled together with the HKL2000 package (Otwinowski and Minor, 1997). The ${ }^{\text {D1D2 }}$ p97-CB-5083 structure was determined by molecular replacement using Protein Data Bank: 3CF2 (Davies et al., 2008) as a search model using the program MOLREP (Vagin and Teplyakov, 2010) in the CCP4 program package (Collaborative Computational Project, Number 4, 1994). The structure was refined using Refmac (Murshudov et al., 1997). The structure model was manually built using the program COOT (Emsley and Cowtan, 2004).

\section{Results and Discussion}

CB-5083 Inhibits ATPase Activities of the FullLength Protein and the D1-D2 Fragment of p97 but Not the N-D1 Fragment. To identify a construct best for crystallizing the p97-CB-5083 complex, we tested three p97 constructs, the full-length protein $\left({ }^{\mathrm{FL}} \mathrm{p} 97\right.$, residues 1-806), an $\mathrm{N}$-D1 fragment $\left({ }^{\mathrm{ND} 1} \mathrm{p} 97\right.$, residues $\left.1-481\right)$, and a D1-D2 fragment $\left({ }^{\mathrm{D} 1 \mathrm{D} 2} \mathrm{p} 97,210-806\right)$. These constructs were expressed and purified, and their ATPase activities were determined in the presence of CB-5083 (Fig. 1, C-E). In the presence of
$2 \mu \mathrm{M} \mathrm{CB}-5083$, both ${ }^{\mathrm{FL}} \mathrm{p} 97$ and ${ }^{\mathrm{D} 1 \mathrm{D} 2} \mathrm{p} 97$ retained $\sim 5 \%$ or less of their original ATPase activity, while that for ${ }^{\mathrm{ND} 1} \mathrm{p} 97$ was nearly unaffected. This result confirmed that the inhibitory action of CB-5083 affects the D2 domain and not the D1 domain. The $\mathrm{IC}_{50}$ values derived from the inhibition assay (Fig. 1; Table 1) also indicated that CB-5083 inhibits ${ }^{\mathrm{D} 1 \mathrm{D} 2} \mathrm{p} 97$ better than ${ }^{\mathrm{FL}} \mathrm{p} 97$, suggesting the ${ }^{\mathrm{D} 1 \mathrm{D} 2} \mathrm{p} 97$ construct would be a better choice for our cocrystallization experiment.

CB-5083 Binds to the ATP-Binding Site at the D2 Domain. Crystals of ${ }^{\mathrm{D} 1 \mathrm{D} 2} \mathrm{p} 97$ in the presence of CB-5083 were successfully obtained and the structure of ${ }^{\mathrm{D} 1 \mathrm{D} 2} \mathrm{p} 97$ in complex with CB-5083 was determined (Table 2). There are 12 independent ${ }^{\text {D1D2 }}$ p97 subunits in a crystallographic asymmetric unit, organizing into two hexameric ${ }^{\mathrm{D} 1 \mathrm{D} 2} \mathrm{p} 97$ rings arranged in a head-to-head fashion (Fig. 2A). After the completion of the model building for the two rings, a difference electron density map was calculated using the Fourier coefficients $m F o-d F c$, which allowed us to look for any ligand bound to the protein. No difference density was observed at any of the 12 D1 nucleotide-binding pockets, demonstrating that the D1 domains are nucleotide or ligand free under the crystallization conditions. This observation is in sharp contrast to previous studies that showed complete nucleotide occupancy of the D1 domain. The bound nucleotides in the D1 ring regulate the $\mathrm{N}$-domain conformation and the D2-domain ATPase activity, and vice versa (Ye et al., 2001; Tang et al., 2010; Niwa et al., 2012). It was also found that a subset of D1 domains of hexameric p97 in a purified protein preparation contains copurified ADP, also called prebound ADP (Davies et al., 2005; Briggs et al., 2008; Tang and Xia, 2013). These prebound ADPs are difficult to remove in wild-type p97 but their binding affinity is reduced in IBMPFD mutants. The lack of bound nucleotides in all of the D1 domains of the current D1-D2 structure suggests that the absence of the $\mathrm{N}$ domain, like the IBMPFD mutants, alters the regulation on the nucleotide state of the D1 domain and hence affects the binding of nucleotide to the D2 domain. In this work, our data show lower $\mathrm{IC}_{50}$ values for the binding of CB-5083 to the D1-D2 fragment, compared with that of wild-type p97. This indicates

TABLE 1

Inhibitory effect of CB-5083 on full-length p97 variants

\begin{tabular}{|c|c|c|c|c|}
\hline P97 Variant & $\mathrm{IC}_{50}^{a}(95 \% \mathrm{CI})^{b}$ & $\mathrm{IC}_{50}$ fold change & Position in Structure & Reference \\
\hline \multicolumn{5}{|c|}{$\mu M$} \\
\hline Wild type ${ }^{c}$ & $0.10(0.08-0.12)$ & - & & This work \\
\hline V474S & $0.88(0.67-1.2)$ & 8.8 & On D1-D2 linker, entrance to ATP site, CB-5083 contacting & This work \\
\hline I479S & $1.2(1.1-1.3)$ & 11.8 & On D1-D2 linker, at ATP site, base and CB-5083 contacting & This work \\
\hline L526S & $1.4(1.3-1.5)$ & 13.9 & At ATP site, base and CB-5083 contacting & This work \\
\hline $\mathrm{I} 656 \mathrm{~S}$ & $0.15(0.13-0.19)$ & 1.5 & At ATP site, base and CB-5083 contacting & This work \\
\hline $\mathrm{D} 1-\mathrm{D} 2^{d}$ & $0.012(0.015-0.02)$ & 0.17 & & This work \\
\hline Wild type ${ }^{c}$ & 0.011 & & & Anderson et al. (2015) \\
\hline N660K & 0.31 & 28.5 & At ATP site, base and CB-5083 contacting & Anderson et al. (2015) \\
\hline A659T & - & & At ATP site, base and CB-5083 contacting & Anderson et al. (2015) \\
\hline T688A & 0.64 & 58 & At ATP site, ribose and CB-5083 contacting & Anderson et al. (2015) \\
\hline V474A & 0.10 & 9.3 & On D1-D2 linker, entrance to ATP site, CB-5083 contacting & Anderson et al. (2015) \\
\hline Q473P & 0.041 & 3.7 & On D1-D2 linker, entrance to ATP site & Anderson et al. (2015) \\
\hline P472L & - & & On D1-D2 linker, entrance to ATP site & Anderson et al. (2015) \\
\hline Wild type ${ }^{c}$ & 0.013 & & & Bastola et al. (2017) \\
\hline E470K & 0.044 & 3.4 & On D1-D2 linker, entrance to ATP site, higher ATPase activity & Bastola et al. (2017) \\
\hline $\mathrm{E} 470 \mathrm{D}$ & 0.049 & 3.8 & On D1-D2 linker, entrance to ATP site, higher ATPase activity & Bastola et al. (2017) \\
\hline
\end{tabular}

CI, confidence interval; - , full length.

${ }^{a}$ The $\mathrm{IC}_{50}$ values in the present study were obtained from three technical replicates and calculated using Prism 7 .

${ }^{b}$ Ninety-five percent confidence level.

${ }^{c}$ The IC I $_{50}$ values from different studies have different ATPase activities due to the use of different ATPase assays.

${ }^{d}$ This is a truncation of p97 containing only D1 and D2 domains. 
TABLE 2

Statistics on the quality of the diffraction data set and refined atomic models

\begin{tabular}{lc}
\hline \multicolumn{1}{c}{ Parameter } & ${ }^{\mathrm{D} 1 \mathrm{p}} \mathrm{p} 97-\mathrm{CB}-5083$ \\
\hline Bound ligand & $\mathrm{CB}-5083$ \\
Data collection & \\
Space group & $P 21$ \\
Unit cell [a, b, c $(\AA)]$ & $127.4,263.8,164.1$ \\
Unit cell $\left[\alpha, \beta, \gamma\left({ }^{\circ}\right)\right]$ & $90.0,103.7,90.0$ \\
Resolution & $50-3.75(3.94-3.75)^{a}$ \\
$R_{\text {merge }}$ & $11.8(77.7)$ \\
Completeness $(\%)$ & $83.9(72.6)$ \\
Total observation & 342,897 \\
Unique reflections & 89,790 \\
$I / \sigma(I)$ & $9.9(1.1)$ \\
$R_{\text {pim }}$ & $0.058(0.500)$ \\
CC $(1 / 2)$ & $0.992(0.239)$ \\
Refinement statistics & \\
Resolution $(\AA)$ & 3.77 \\
$R_{\text {free }}(\%)$ & 26.5 \\
$R_{\text {work }}(\%)$ & 22.2 \\
$R_{\text {msd }}$ bond length $(\AA)$ & 0.008 \\
$R_{\text {msd }}$ bond angle $\left({ }^{\circ}\right)$ & 1.289 \\
Coordinate error $(\AA)$ & 0.729 \\
Number of non-H atoms & 48,440 \\
Number of residues & 6152 \\
Number of solvent atoms & 33 \\
Number of CB-5083 & 12 \\
Ramachandran analysis & \\
Most favored (\%) & 87.4 \\
Allowed (\%) & 12.6 \\
Disallowed $(\%)$ & 0 \\
PDB code & $6 \mathrm{MCK}$ \\
\hline
\end{tabular}

CC (1/2), correlation coefficient between intensities from random half-datasets; PDB, Protein Data Bank; $R_{m s d}$, root mean square deviation.

${ }^{a}$ Values in parentheses are for the highest resolution shells.

${ }^{b} R_{\text {merge }}$ is defined as $\Sigma\left|\mathbf{I}_{\mathbf{h}, \mathbf{i}}-<\mathbf{I}_{\mathbf{h}}>\right| \Sigma \mathbf{I}_{\mathbf{h}, \mathbf{i}}$, where $\mathbf{I}_{\mathbf{h}, \mathbf{i}}$ is the intensity for ith observation of a reflection with Miller index $\mathbf{h}$, and $<\mathbf{I}_{\mathbf{h}}>$ is the mean intensity for all measured $\mathbf{I}_{\mathbf{h}}$ s and Friedel pairs.

that removing the $\mathrm{N}$ domains leads to an empty D1 domain, which in turn leads to a lowered $\mathrm{IC}_{50}$ value for $\mathrm{CB}-5083$ binding. This sequence of events is similar to IBMPFD mutant-induced higher ATPase activity at the D2 domain (Halawani et al., 2009; Manno et al., 2010; Niwa et al., 2012; Tang and Xia, 2013).

On the contrary, large positive densities were observed at the nucleotide-binding pockets of all 12 D2 domains (Fig. 2B). These densities have a shape and size resembling those of CB-5083 and were modeled with one molecule of CB-5083 for each domain. Subsequently, the model for the ${ }^{\mathrm{D} 1 \mathrm{D} 2} \mathrm{p} 97-\mathrm{CB}-$ 5083 complex was refined to a resolution of $3.77 \AA$, with $R_{\text {work }}$ and $R_{\text {free }}$ values of 22.2 and 26.5 , respectively (Table 2 ).

A molecule of CB-5083 intercalates into part of the D2 ATPbinding pocket, occupying the space for the adenine base and ribose moiety of the nucleotide (Fig. 2C). The binding energy is clearly derived from both hydrogen-bonding and hydrophobic interactions (Fig. 2B). The benzylamino moiety of CB-5083 penetrates deep into the pocket, occupying a hydrophobic niche, in direct contact with five hydrophobic residues (I479, L482, L526, L527, and I656). The CB-5083 molecule is further stabilized by a strong hydrogen bond (2.5-3 $\AA$ ) between the hydroxy group of residue T688 and the oxygen atom of the amide side chain of the carbamoyl-indole moiety (Fig. $2 \mathrm{~B})$. The orientation of the carbamoyl-indole moiety appears to be maintained by N660, which also forms a hydrogen bond with T688 (Fig. 2B). The dihydro-pyrano-pyrimidine core of CB-5083 is wedged between L526 and V474 on the one side and $\mathrm{A} 659$ on the other side.
Since the bound CB-5083 occupies to a large extent the same binding pocket as that for bound ATP at the D2 domain, we wondered if the observed positive difference density could possibly come from a bound nucleotide, although we did not include nucleotides in any of the experiments. We, therefore, superposed two D2 domains, one with CB-5083 bound and the other with adenylyl-imidodiphosphate (AMP-PNP) bound (Protein Data Bank: 3CF2) (Fig. 2C). The superposition shows that only the carbamoyl-indole moiety of the bound CB-5083 partially overlaps with the adenine and ribose moieties of AMP-PNP. There is no extra density in the CB-5083 structure corresponding to the phosphate groups of AMP-PNP, and the dihydro-pyrano-pyrimidine core and benzylamino moiety of CB-5083 do not correspond to any part of the nucleotide. In addition, the shape and size of the difference density do not fit well with the adenine and ribose moieties of AMP-PNP in standard geometry. Therefore, we are confident that the difference density belongs to the bound CB-5083.

The Experimental Model Is Supported by StructureActivity Relationship and Mutagenesis Studies. The structure of the p97-CB-5083 complex explains the following structure-activity relationship observations (Zhou et al., 2015): 1) no methyl group attaching to the nitrogen atom [- $\mathrm{N}\left(\mathrm{CH}_{3}\right) \mathrm{CH}_{2} \mathrm{Ph}$ in the benzylamino group can be tolerated; 2) no extra $\mathrm{CH}_{2}$ groups can be inserted into the benzylamino group ( $-\mathrm{NHCH}_{2} \mathrm{CH}_{2} \mathrm{Ph}$ or $-\mathrm{NHCH}_{2} \mathrm{CH}_{2} \mathrm{CH}_{2} \mathrm{Ph}$ ); 3 ) a hydrophilic group in place of the methyl group in the carbamoylindole moiety of CB-5083 is detrimental to binding affinity; and 4) no group can be tolerated at the fifth position of the carbamoyl-indole moiety.

The structure is also consistent with many key resistant mutants described in the literature (Table 1). 1) The T688A mutation led to a 58 -fold increase in the $\mathrm{IC}_{50}$ value because of the loss of the critical hydrogen bond. 2) The N660K mutation led to a 29 -fold increase in the $\mathrm{IC}_{50}$ value due to a broken hydrogen bond to the $-\mathrm{OH}$ group of the nearby residue $\mathrm{T} 688$. Also, the larger side chain of the lysine residue possibly destabilizes the orientation of the carbamoyl-indole moiety of CB-5083. 3) The five hydrophobic residues (I479, L482, L526, L527, and I656) form a hydrophobic niche and are in contact with the benzylamino group. We introduced three mutations to this niche, I479S, L526S, and I656S, individually, turning it into a more hydrophilic pocket. Except for the I656S mutation, either the I479S or L526S mutation led to $\sim 10$-fold larger increase in the $\mathrm{IC}_{50}$ value. 4) Similarly, V474 is involved in contact with the dihydro-pyrano-pyrimidine core of CB-5083 and the resistance mutation V474A led to a 10-fold increase in the $\mathrm{IC}_{50}$ value, which was confirmed by a mutation (V474S) we also introduced that led to a similar enlargement in the $\mathrm{IC}_{50}$ value (Table 1 ).

Some Mutations that Confer Resistance to CB-5083 Are Found in the Entrance Portal to the ATP-Binding Site. A number of mutations conferring resistance to CB-5083, which are not in direct contact with the bound inhibitor, have been identified, but were difficult to interpret in the absence of an experimental model (Table 1). A common characteristic of these mutations is the smaller increase in the $\mathrm{IC}_{50}$ values in CB-5083 binding. These mutations $(\mathrm{E} 470 \mathrm{~K} / \mathrm{D}$, P472L, Q473P, and V474A) were found to be localized at the D1D2 linker region (residues 460-481) and shown to alter the ATPase activities (Anderson et al., 2015; Bastola et al., 2017). Interestingly, the mutated residues are all clustered at the 
A
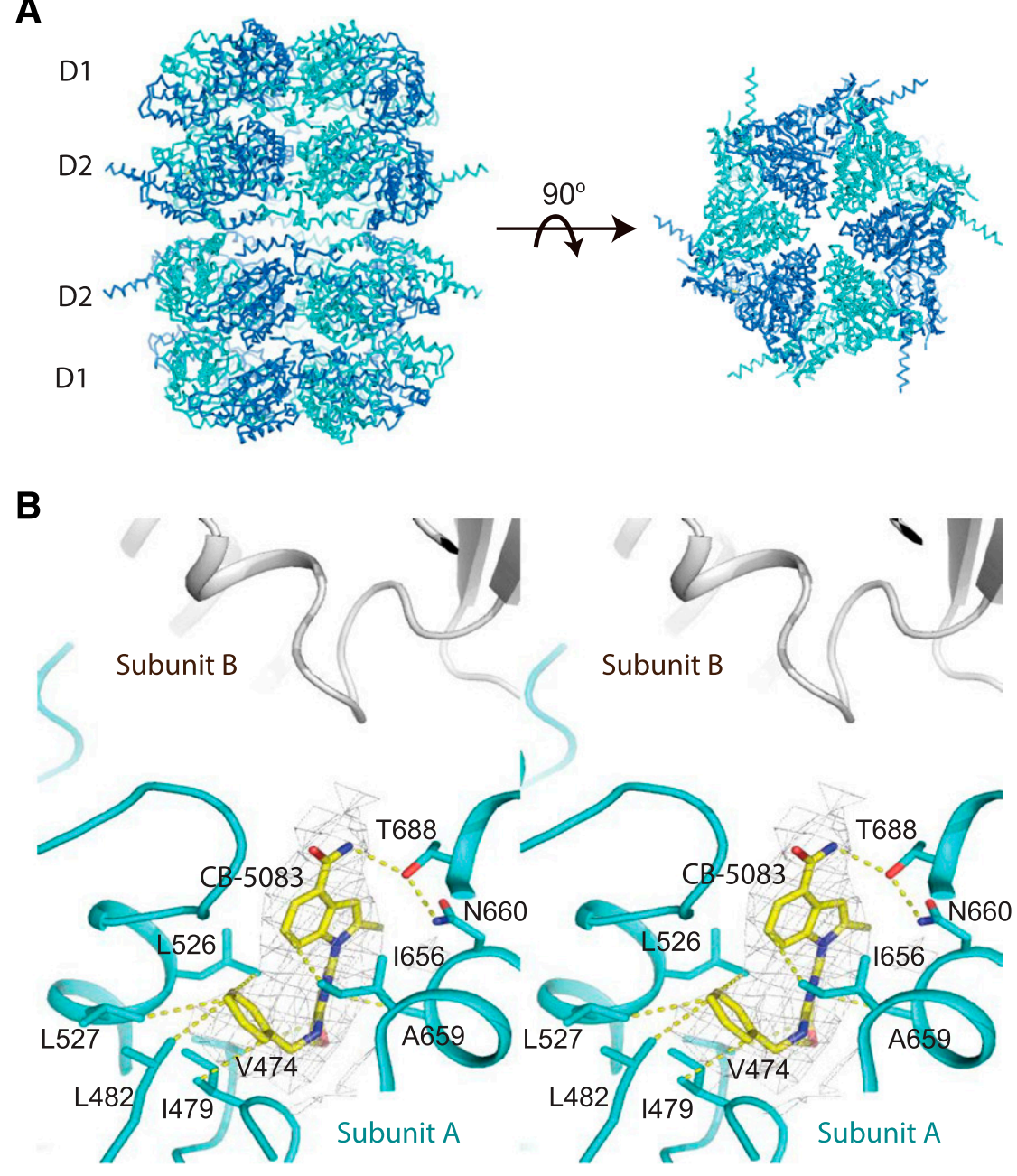

C

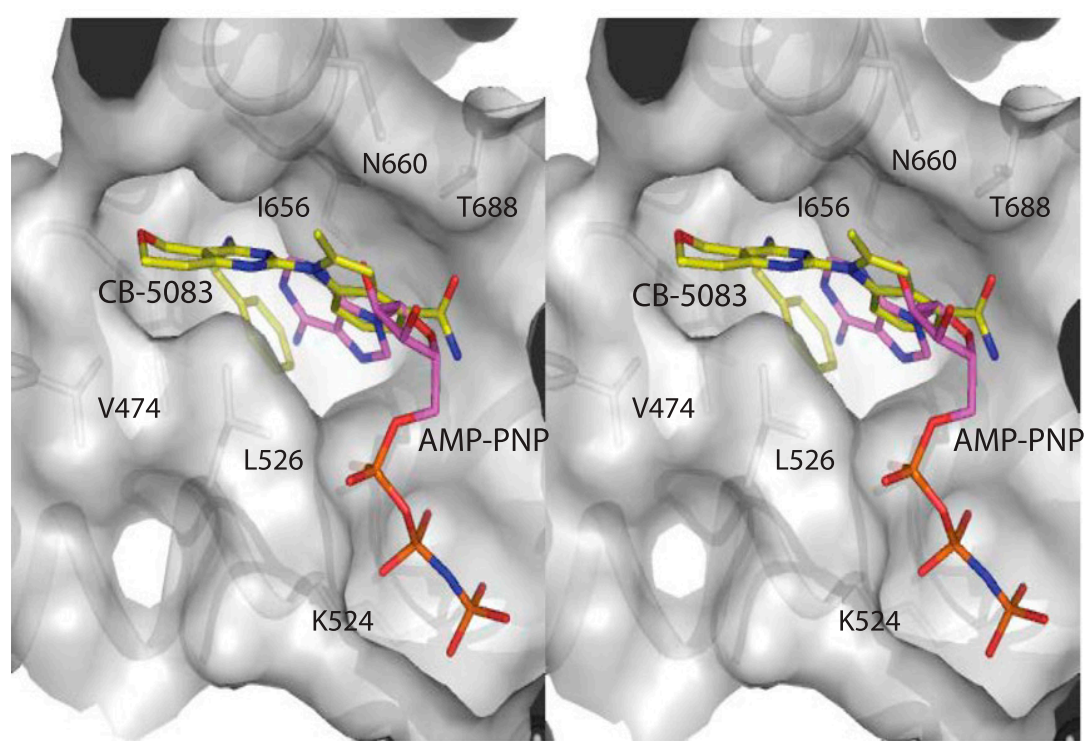

Fig. 2. Interactions between p97 and CB-5083. (A) The content in the crystallographic asymmetric unit containing two ${ }^{\mathrm{D} 1 \mathrm{D} 2} \mathrm{p} 97$ hexameric rings arranged in a head-to-head fashion is shown for a crystal of ${ }^{\mathrm{D} 1 \mathrm{D} 2} \mathrm{p} 97$ in complex with CB-5083. The side and top views of the rings are given. The subunits are alternately colored in blue and cyan. (B) Stereoscopic pair showing details of the CB-5083 binding environment. The CB-5083 bound at the D2 nucleotide-binding pocket is shown as a stick model with carbon, oxygen, and nitrogen atoms colored in yellow, red, and blue, respectively. The difference electron density for CB-5083, shown as a gray wire cage and contoured at the $3 \sigma$ level, is calculated using the Fourier coefficients of $m F o-d F c$, where $F c$ was obtained with CB-5083 omitted. hydrogen bond interactions and close contacts are shown in dotted lines. One subunit of ${ }^{\mathrm{D} 1 \mathrm{D} 2} \mathrm{p} 97$ is colored in cyan and the neighboring one in gray. Residues involved in contacting with CB-5083 are shown as stick models with labels. (C) Stereo pair showing the superposition of two D2 domains, one with CB-5083 bound and the other with AMP-PNP bound (Protein Data Bank: 3CF2). The CB-5083 and AMP-PNP molecules are shown as stick models with carbon, oxygen, nitrogen, and phosphorous atoms in yellow (CB-5083)/magenta (AMP-PNP), red, blue, and orange, respectively. The D2 domain is shown as a semitransparent molecular surface with interacting residues rendered as stick models with labels. 


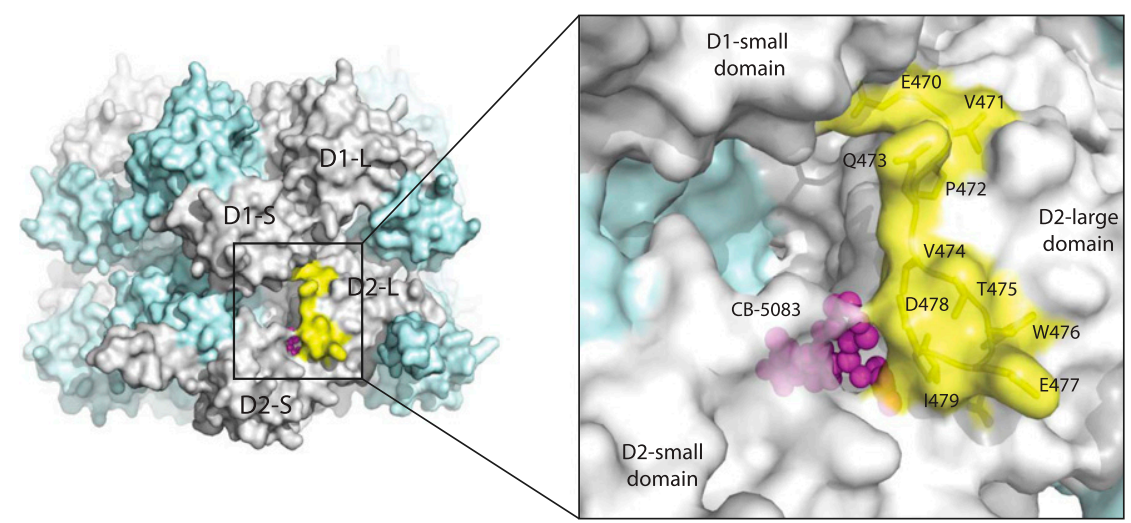

Fig. 3. Environment of the D1D2 linker region (residues 463-483). The hexameric D1D2 fragment of p97 is depicted as a molecular surface with alternating gray and cyan subunits. Each AAA domain has a large domain (D1-L and D2-L) and a small domain (D1-S and D2-S). The bound CB-5083 is shown as a ball model in magenta and its binding pocket is at the interface of the two subdomains (D2-L and D2-S) of the D2 domain. The binding site also shares a border with a neighboring subunit. The D1D2 linker is shown as a yellow molecular surface. The right panel is the zoom-in image showing the CB-5083 binding pocket and the D1D2 linker. The D1D2 linker region is presented as a cartoon overlayed with a semitransparent surface in yellow with residues that are involved in resistance rendered as stick models and labeled. entrance leading to the nucleotide-binding pocket (Fig. 3). Thus, mutations of these residues may affect the accessibility of CB-5083 to the binding site.

Structural Difference in D1 Nucleotide-Binding Pocket Precludes Its Binding of CB-5083. The two ATPase domains D1 (210-460) and D2 (481-760) of p97 share $\sim 40 \%$ and $\sim 60 \%$ sequence identity and similarity, respectively. Both domains are capable of binding and hydrolyzing ATP. Despite these similarities, biochemical and structural data showed CB-5083 binding specifically to the ATP-binding pocket of the D2 domain. To reveal the molecular details that define the binding preference of CB-5083 for the D2 domain, we looked at two possibilities for the binding difference: access of the drug to the sites and difference in molecular shapes of the two sites. The ATP-binding site of the D1 domain is accessible from either the top (Fig. 4A) or the side of the hexamer (data not shown). While the side entrance can be blocked by the movement of the $\mathrm{N}$ domain, the one on the top remains unchanged during the D1 ATPase cycle. Thus, it appears unlikely that access of CB-5083 to the D1 site is the cause for the drug's preference for the D2 domain. The D2 domain, on the other hand, has only one access portal to the ATP-binding site, which is on the side of the hexamer, and the shape can be modulated by the D1D2 linker (Fig. 4B). The ATP site is less accessible from the bottom of the molecule.

To examine the molecular shape of the two sites, we superposed the two ATPase domains and compared their binding pockets (Fig. 4C). From the structure of the complex
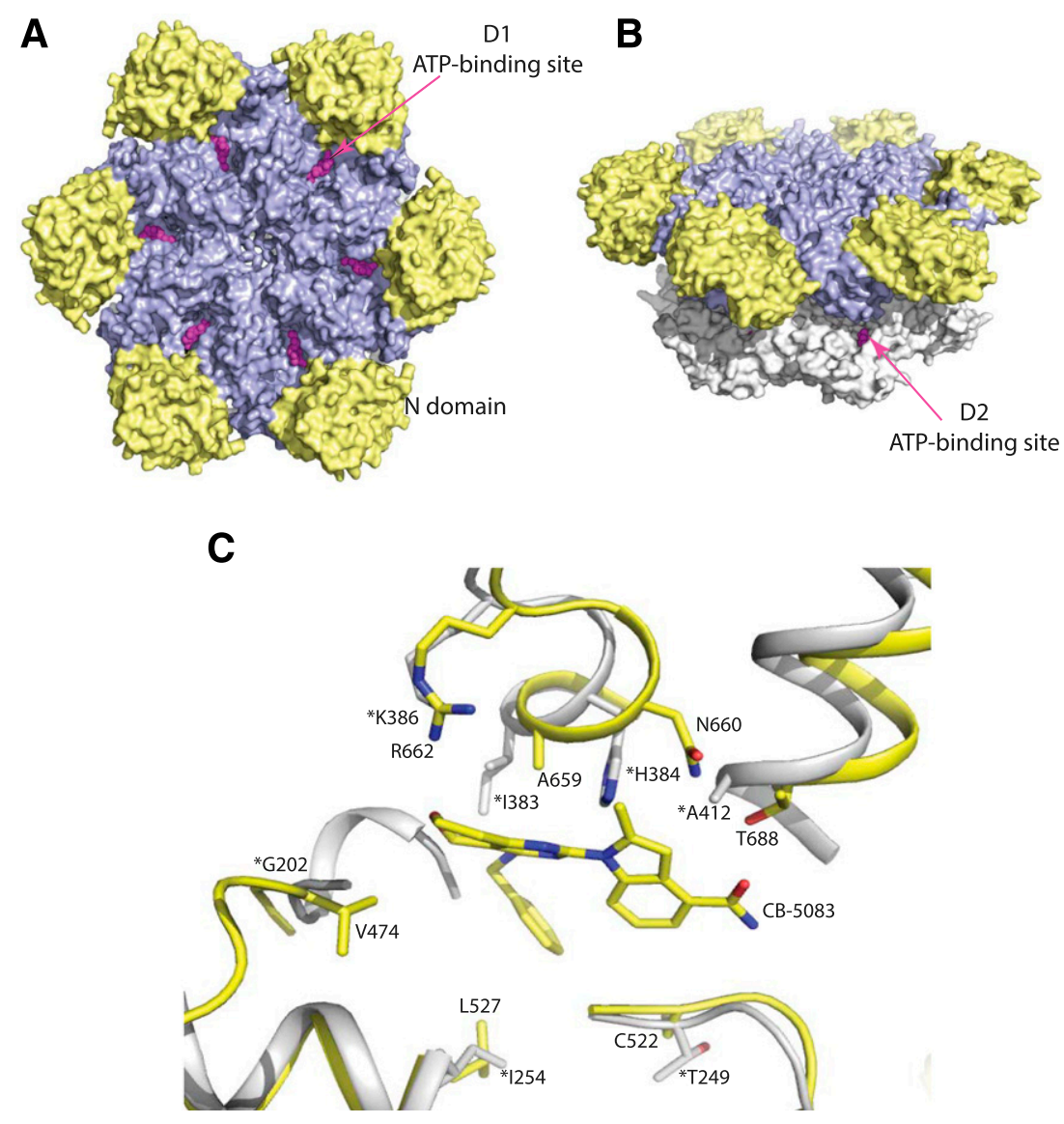

Fig. 4. Molecular difference between the D1 and D2 ATP-binding sites of p97. (A) Top view of the full-length p97 (Protein Data Bank: 3CF3) hexamer in a surface representation showing the D1 ATP-binding sites occupied by $\mathrm{ADP}$ rendered as magenta spheres. The $\mathrm{N}$ and $\mathrm{D} 1$ domains are colored yellow and light blue, respectively. (B) Side view of the full-length p97 hexameric ring showing the D2 ATP-binding site occupied by ADP, shown as magenta spheres. The D2 domain is in gray. (C) Cartoon representation showing the ATP-binding pocket with D1 and D2 domains superposed. Residues that are in close contact with CB-5083 are rendered as sticks and labeled. The corresponding residues in the D1 domain are also rendered as sticks and labeled with asterisks. 
TABLE 3

D2-domain CB-5083 contacting residues that are different in corresponding residues in the D1 domain

\begin{tabular}{lll}
\hline D2 Residue & D1 Residue & \multicolumn{1}{c}{ Function for CB-5083 Binding } \\
\hline L527 & I254 & Contact benzylamino group \\
L482 & C209 & Contact benzylamino group \\
C522 & T249 & Contact benzylamino group \\
T688 & A412 & Contact carbamoyl-indole moiety \\
N660 & H384 & Contact carbamoyl-indole moiety \\
R662 & K386 & Contact dihydro-pyrano-pyrimidine core \\
A659 & I383 & Contact dihydro-pyrano-pyrimidine core \\
V474 & G202 & Contact dihydro-pyrano-pyrimidine core \\
\hline
\end{tabular}

and the resistant mutations, we learned that several residues are important for interacting with and stabilizing CB-5083 bound at the D2 domain. These residues include T688 and N660 because mutations to these two residues cause 58- and 29-fold increases in the $\mathrm{IC}_{50}$ values, respectively, for CB-5083 binding (Fig. 2C; Table 1). The superposition shows that the residues in the D1 domain corresponding to T688 and N660 of the D2 domain are A412 and H384 (Fig. 4C), which are unable to form the hydrogen bonds essential for CB-5083 binding. Many D2 site residues that contact CB-5083 are not conserved for the D1 site (Table 3), indicating that the differences in size and shape between the D1 and D2 sites are sufficiently large to favor CB-5083 binding to the D2 site.

Furthermore, some residues of the D1D2 linker, such as V474, I479, and G480, are in direct contact with the bound CB-5083 (Fig. 3), being an integral part of the CB-5083binding pocket. Indeed, mutations in these residues altered the sensitivity of p97 to CB-5083 (Table 1) (Anderson et al., 2015). This feature of the CB-5083-binding pocket of the D2 domain has no equivalence for the ATP-binding pocket of the D1 domain, although it may be approximated by the ND1 linker (residues 180-200), which has shown a large positional deviation from the D1D2 linker in the structural alignment. Moreover, the ND1 linker is conformationally variable depending upon the nucleotide states at the D1 domain, undergoing a helix-to-loop conformational switch (Tang et al., 2010) and thus introducing a dramatic change to the shape and size of the D1 ATP site.

Summary. A recent update on the clinical trials of CB-5083, reported in the San Francisco Business Times, revealed an off-target effect of the drug interacting with phosphodiesterase- 6 , a protein involved in phototransduction in the eyes. While the clinical trials of CB-5083 have been halted for the safety of patients, the idea of targeting p97 for cancer therapy remains valid. To circumvent the off-target side effect of CB-5083, a new generation of drugs, preferably based on CB-5083, with enhanced specificity, is being developed by a multi-institutional consortium. Such an effort is best served with an accurate atomic model describing accurately the interactions of p97 with CB-5083. The significance of this work lies not only in providing an experimental model for the interaction between CB-5083 and p97 but also in offering guidance on how to modify the drug for better affinity and specificity. Such structure-based drug improvement has been shown to be most effective when an accurate model is available (Hao et al., 2012; Lounnas et al., 2013).

One suggestion is to modify the benzylamino moiety of CB-5083, which fits into the hydrophobic niche of the D2 nucleotide-binding site. At the end of this hydrophobic niche is the residue C522. A modification could be made to the benzylamino moiety such that it is able to form a covalent bond with C522, which could potentially increase the efficacy and perhaps the specificity of CB-5083. Also, R662 and D478 are located within $4 \AA$ of the dihydro-pyrano-pyrimidine core. Alterations introduced to this core could potentially introduce salt bridges between the drug and p97, further enhancing existing interactions.

\section{Acknowledgments}

We thank the staff members of the SER-CAT and GM/CA beamlines at the Advanced Photon Source, Argonne National Laboratory (Lemont, IL), for assistance in data collection. All DNA sequencing services were conducted at the Center for Cancer Research Genomics Core at the National Cancer Institute, National Institutes of Health (Bethesda, MD). We also thank George Leiman for editorial assistance.

\section{Authorship Contributions}

Participated in research design: Tang, Xia.

Conducted experiments: Tang, Odzorig, Jin.

Performed data analysis: Tang, Xia.

Wrote or contributed to the writing of the manuscript: Tang, Xia.

\section{References}

Anderson DJ, Le Moigne R, Djakovic S, Kumar B, Rice J, Wong S, Wang J, Yao B, Valle E, Kiss von Soly S, et al. (2015) Targeting the AAA ATPase p97 as an approach to treat cancer through disruption of protein homeostasis. Cancer Cell 28: 653-665.

Bastola P, Wang F, Schaich MA, Gan T, Freudenthal BD, Chou TF, and Chien J (2017) Specific mutations in the D1-D2 linker region of VCP/p97 enhance ATPase activity and confer resistance to VCP inhibitors. Cell Death Discov 3:17065.

Beskow A, Grimberg KB, Bott LC, Salomons FA, Dantuma NP, and Young P (2009) A conserved unfoldase activity for the p97 AAA-ATPase in proteasomal degradation. $J$ Mol Biol 394:732-746.

Blythe EE, Olson KC, Chau V, and Deshaies RJ (2017) Ubiquitin- and ATPdependent unfoldase activity of P97/VCP•NPLOC4•UFD1L is enhanced by a mutation that causes multisystem proteinopathy. Proc Natl Acad Sci USA 114 E4380-E4388.

Bodnar NO and Rapoport TA (2017) Molecular mechanism of substrate processing by the Cdc48 ATPase complex. Cell 169:722-735.e9.

Briggs LC, Baldwin GS, Miyata N, Kondo H, Zhang X, and Freemont PS (2008) Analysis of nucleotide binding to P97 reveals the properties of a tandem AAA hexameric ATPase. $J$ Biol Chem 283:13745-13752.

Chou TF and Deshaies RJ (2011) Quantitative cell-based protein degradation assays to identify and classify drugs that target the ubiquitin-proteasome system. J Biol Chem 286:16546-16554.

Chou TF, Li K, Frankowski KJ, Schoenen FJ, and Deshaies RJ (2013) Structureactivity relationship study reveals ML240 and ML241 as potent and selective inhibitors of p97 ATPase. ChemMedChem 8:297-312.

Collaborative Computational Project, Number 4 (1994) The CCP4 suite: programs for protein crystallography. Acta Crystallogr D Biol Crystallogr 50:760-763.

Davies JM, Brunger AT, and Weis WI (2008) Improved structures of full-length p97, an AAA ATPase: implications for mechanisms of nucleotide-dependent conformational change. Structure 16:715-726.

Davies JM, Tsuruta H, May AP, and Weis WI (2005) Conformational changes of p97 during nucleotide hydrolysis determined by small-angle X-ray scattering. Structure 13:183-195.

Emsley P and Cowtan K (2004) Coot: model-building tools for molecular graphics. Acta Crystallogr D Biol Crystallogr 60:2126-2132.

Gareau A, Rico C, Boerboom D, and Nadeau ME (2018) In vitro efficacy of a firstgeneration valosin-containing protein inhibitor (CB-5083) against canine lymphoma. Vet Comp Oncol 16:311-317.

Ghosh R, Wang L, Wang ES, Perera BG, Igbaria A, Morita S, Prado K, Thamsen M, Caswell D, Macias H, et al. (2014) Allosteric inhibition of the IRE1 $\alpha$ RNase preserves cell viability and function during endoplasmic reticulum stress. Cell 158: $534-548$

Gugliotta G, Sudo M, Cao Q, Lin DC, Sun H, Takao S, Le Moigne R, Rolfe M, Gery S, Müschen M, et al. (2017) Valosin-containing protein/p97 as a novel therapeutic target in acute lymphoblastic leukemia. Neoplasia 19:750-761.

Halawani D, LeBlanc AC, Rouiller I, Michnick SW, Servant MJ, and Latterich M (2009) Hereditary inclusion body myopathy-linked p97/VCP mutations in the NH2 domain and the D1 ring modulate p97/VCP ATPase activity and D2 ring conformation. Mol Cell Biol 29:4484-4494.

Hao GF, Wang F, Li H, Zhu XL, Yang WC, Huang LS, Wu JW, Berry EA, and Yang GF (2012) Computational discovery of picomolar $Q_{\mathrm{o}}$ site inhibitors of cytochrome bc1 complex. J Am Chem Soc 134:11168-11176.

Hess HH and Derr JE (1975) Assay of inorganic and organic phosphorus in the 0.1-5 nanomole range. Anal Biochem 63:607-613.

Kale AJ and Moore BS (2012) Molecular mechanisms of acquired proteasome inhibitor resistance. J Med Chem 55:10317-10327. 
Kenny TC, Manfredi G, and Germain D (2017) The mitochondrial unfolded protein response as a non-oncogene addiction to support adaptation to stress during transformation in cancer and beyond. Front Oncol 7:159.

Lanzetta PA, Alvarez LJ, Reinach PS, and Candia OA (1979) An improved assay for nanomole amounts of inorganic phosphate. Anal Biochem 100:95-97.

Le Moigne R, Aftab BT, Djakovic S, Dhimolea E, Valle E, Murnane M, King EM, Soriano F, Menon MK, Wu ZY, et al. (2017) The p97 inhibitor CB-5083 is a unique disrupter of protein homeostasis in models of multiple myeloma. Mol Cancer Ther 16:2375-2386

Lounnas V, Ritschel T, Kelder J, McGuire R, Bywater RP, and Foloppe N (2013) Current progress in structure-based rational drug design marks a new mindset in drug discovery. Comput Struct Biotechnol J 5:e201302011.

Manno A, Noguchi M, Fukushi J, Motohashi Y, and Kakizuka A (2010) Enhanced ATPase activities as a primary defect of mutant valosin-containing proteins that cause inclusion body myopathy associated with Paget disease of bone and frontotemporal dementia. Genes Cells 15:911-922.

Milano A, Perri F, and Caponigro F (2009) The ubiquitin-proteasome system as a molecular target in solid tumors: an update on bortezomib. OncoTargets Ther 2: 171-178.

Murshudov GN, Vagin AA, and Dodson EJ (1997) Refinement of macromolecular structures by the maximum-likelihood method. Acta Crystallogr D Biol Crystallogr 53:240-255.

Niwa H, Ewens CA, Tsang C, Yeung HO, Zhang X, and Freemont PS (2012) The role of the N-domain in the ATPase activity of the mammalian AAA ATPase p97/VCP. $J$ Biol Chem 287:8561-8570.

Otwinowski Z and Minor W (1997) Processing of X-ray diffraction data collected in oscillation mode. Methods Enzymol 276:307-326.

Scalzulli E, Grammatico S, Vozella F, and Petrucci MT (2018) Proteasome inhibitors for the treatment of multiple myeloma. Expert Opin Pharmacother 19:375-386.

Tang WK, Li D, Li CC, Esser L, Dai R, Guo L, and Xia D (2010) A novel ATP dependent conformation in p97 N-D1 fragment revealed by crystal structures of disease-related mutants. EMBO J 29:2217-2229.
Tang WK and Xia D (2013) Altered intersubunit communication is the molecular basis for functional defects of pathogenic p97 mutants. J Biol Chem 288: 36624-36635.

Tang WK and Xia D (2016) Mutations in the human $\mathrm{AAA}^{+}$chaperone p97 and related diseases. Front Mol Biosci 3:79.

Taylor JP (2015) Multisystem proteinopathy: intersecting genetics in muscle, bone, and brain degeneration. Neurology 85:658-660.

Vagin A and Teplyakov A (2010) Molecular replacement with MOLREP. Acta Crystallogr D Biol Crystallogr 66:22-25.

Wang Q, Li L, and Ye Y (2008) Inhibition of p97-dependent protein degradation by Eeyarestatin I. J Biol Chem 283:7445-7454.

Wang Q, Mora-Jensen H, Weniger MA, Perez-Galan P, Wolford C, Hai T, Ron D, Chen W, Trenkle W, Wiestner A, et al. (2009) ERAD inhibitors integrate ER stress with an epigenetic mechanism to activate BH3-only protein NOXA in cancer cells. Proc Natl Acad Sci USA 106:2200-2205.

Xia D, Tang WK, and Ye Y (2016) Structure and function of the AAA+ ATPase p97/Cdc48p. Gene 583:64-77.

Ye Y, Meyer HH, and Rapoport TA (2001) The AAA ATPase Cdc48/p97 and its partners transport proteins from the ER into the cytosol. Nature 414:652-656.

Ye Y, Meyer HH, and Rapoport TA (2003) Function of the p97-Ufd1-Npl4 complex in retrotranslocation from the ER to the cytosol: dual recognition of nonubiquitinated polypeptide segments and polyubiquitin chains. J Cell Biol 162:71-84.

Zhou HJ, Wang J, Yao B, Wong S, Djakovic S, Kumar B, Rice J, Valle E, Soriano F, Menon MK et al. (2015) Discovery of a first-in-class, potent, selective, and orally bioavailable inhibitor of the p97 AAA ATPase (CB-5083). J Med Chem 58:9480-9497.

Address correspondence to: Di Xia, Laboratory of Cell Biology, Center for Cancer Research, National Cancer Institute, National Institutes of Health, Bethesda, 37 Convent Drive, Room 2122C, Bethesda, MD 20892. E-mail: xiad@ mail.nih.gov 